\title{
Reliabilität und Validität des MoMo-Aktivitätsfragebogens für Jugendliche (MoMo-AFB)
}

\author{
Darko Jekauc, Matthias O. Wagner, \\ Daniela Kahlert und Alexander Woll
}

\begin{abstract}
Zusammenfassung. Der vorliegende Beitrag beschreibt die psychometrischen Eigenschaften des Aktivitätsfragebogens des Motorikmoduls (MoMo-AFB; Bös, Worth, Opper, Oberger \& Woll, 2009), einer Skala zur Messung körperlicher Aktivität bei Kindern und Jugendlichen. Der Fragebogen besteht aus 28 Items, die Alltagsaktivität, Sport in und außerhalb von organisierten Vereinen, Schulsport und die Erfüllung von Aktivitätsguidelines erfassen. Untersucht wurden 109 Schüler und 87 Schülerinnen im Alter zwischen 9 und 17 Jahren. Zur Überprüfung der Reliabilität wurde der Fragebogen zweimal in einem Abstand von sieben Tagen ausgefüllt. Zur Untersuchung der kriteriumsbezogenen Validität wurden die Fragebogendaten mit Accelerometerdaten (ActiGraph GT1X) verglichen. Der durchschnittliche (gewichtete) Kappa-Koeffizient und die Intraklassenkorrelation (ICC) für den Gesamtindex betrugen .66 bzw .68. Der mittels MoMo-AFB und Accelerometer ermittelte Gesamtumfang der Zeit mit moderater bis hoher Intensität der KA zeigte eine signifikante Spearman-Korrelation von $r \quad$.29. Der MoMo-AFB ist bezüglich der Reliabilität und Validität vergleichbar mit anderen international publizierten Aktivitätsfragebögen für Jugendliche.

Schlüsselwörter: Körperliche Aktivität, Aktivitätsfragebögen, MoMo-AFB, Jugendliche
\end{abstract}

Reliability and validity of MoMo-Physical-Activity-Questionnaire for Adolescents (MoMo-AFB)

\begin{abstract}
This paper presents the psychometric properties of the MoMo-AFB (Bös, Worth, Opper, Oberger \& Woll, 2009), a questionnaire designed to measure the physical activity of children and adolescents. The MoMo-AFB consists of 28 items, covering the fields of everyday physical activity, sports within and outside of organised clubs, physical education as well as compliance with physical activity guidelines. A study was conducted with 109 boys and 87 girls aged between 9 and 17 years. The MoMo-AFB was given on two separate occasions to evaluate reliability and was compared with accelerometry data of ActiGraph GT1X to evaluate validity. The overall kappa/weighted kappa coefficient for the one-week testretest reliability was .66 ( $S D \quad .19)$ and the overall intraclass correlation (ICC) .68. Self-reported and accelerometer-measured average weekly time spent performing moderate to vigorous physical activity were significantly correlated (Spearman $r \quad$.29). Regarding reliability and validity, the MoMo-AFB is comparable with other international published physical activity questionnaires for adolescents.

Key words: physical activity, physical activity questionnaire, MoMo-AFB, adolescents
\end{abstract}

Körperliche Inaktivität wurde als viertgrößter Mortalitätsrisikofaktor nach Bluthochdruck, Rauchen und erhöhten Blutzuckerwerten identifiziert (WHO, 2010). Durch regelmäßige körperliche Aktivität (KA) konnte bei Erwachsenen mehrfach ein niedrigeres Risiko für verschiedene Krankheiten, wie beispielsweise Erkrankungen des Herz-Kreislauf-Systems, Typ-2-Diabetes-melitus, Übergewicht, Bluthochdruck, Osteoporose, Depression, Angststörungen u.a. festgestellt werden (vgl. im Überblick Dishman, Washburn \& Heath, 2004). Die Ergebnisse eines Reviews von 850 Beiträgen für das Kindes- und Jugendalter zeigen, dass regelmäßige KA notwendig ist, um eine normale physische (z.B. Motorik) und psychische (z.B. globaler Selbstwert, Selbstkonzept, Wohlbefinden usw.) Entwicklung zu gewährleisten (vgl. Strong et al., 2005). Aufgrund der Tatsache, dass es kaum längsschnittliche Studien mit Kindern und Jugendlichen gibt, ist der Zusammenhang zwischen KA und
Gesundheit für diese Altersgruppen allerdings weniger eindeutig (siehe auch Völker, 2009), gleichwohl wurden positive Effekte auf den globalen Selbstwert, das Selbstkonzept und das Wohlbefinden diskutiert (West Suitor \& Kraak, 2007). Eine wichtige Voraussetzung, um den Zusammenhang zwischen KA und Gesundheit untersuchen zu können, ist die adäquate Messung der KA. Ziel der vorliegenden Studie ist daher die Prüfung der Reliabilität und Validität eines Fragebogens zur Erfassung der KA bei Jugendlichen.

\section{Zum Konzept der körperlichen Aktivität}

Unter KA versteht man alle körperlichen Bewegungen, die durch die Skelettmuskulatur hervorgerufen werden und 
einen substantiellen Energieverbrauch zur Folge haben (Caspersen, Powell \& Christensen, 1985). Dies schließt den Weg zur Schule, Hausarbeiten oder Freizeitaktivitäten ebenso wie spielerische und sportliche Aktivitäten ein. Ausgegrenzt sind bei dieser Definition körperinterne Bewegungen (z.B. Darmaktivität) sowie spielerisch-sportliche Betätigungen ohne wesentlichen Energieverbrauch (z. B. Schachspielen).

Die Quantifizierung der KA bei Kindern und Jugendlichen unter alltäglichen Bedingungen gestaltet sich kompliziert, da sich die Aktivitätseinheiten meist in unterschiedlichen Mengen, mit unterschiedlicher Intensität und unregelmäßig über den Tag verteilen. Ferner kann man KA als ein komplexes Verhalten mit mehreren Dimensionen und Domänen charakterisieren, das mit unterschiedlicher Regelmäßigkeit verfolgt wird (vgl. Trost, 2007). Zu den Dimensionen der KA werden Dauer, Häufigkeit, Intensität und Art der KA gezählt. Vor dem Hintergrund der theoretischen Überlegungen zur Zeitstruktur der kindlichen Welt (Neumann, 1997), ist speziell für die Messung der KA bei Kindern und Jugendlichen eine settingbezogene Unterteilung der Domänen der KA sinnvoll. Bei dieser Einteilung handelt es sich um eine Konvention, die sich in der interdisziplinären Aktivitätsforschung durchgesetzt hat (Trost, 2007). Bei Kindern und Jugendlichen sind dies die Bereiche der KA in der Schule (z.B. Schulsport), Freizeitsport in Vereinen (z.B. Volleyballtraining), Freizeitsport außerhalb von Vereinen (z.B. Fußball mit Freunden auf der Wiese) und Alltagsaktivitäten (z. B. Weg zur Schule, Einkaufen etc.). Daher sollten Instrumente zur Erfassung der KA eine reliable und valide Abbildung der vier Dimensionen in allen Domänen gewährleisten.

Schließlich wird zwischen aktueller und habitueller KA unterschieden. Während sich aktuelle KA auf einen relativ kurzen Zeitraum (z. B. einen Tag oder eine Woche) bezieht, ist bei habitueller KA der Erfassungszeitraum (z. B. mehrere Monate oder ein Jahr) wesentlich größer. In der gesundheitspsychologischen Forschung wird vor allem die Bedeutung von habituellen Verhaltensweisen für die Vorhersage des Gesundheitszustandes betont (vgl. z.B. Becker, Bös, Opper, Woll \& Wustmans, 1996), weshalb diese bei der Erfassung der KA besondere Beachtung finden sollten.

\section{Methoden zur Erfassung der körperlichen Aktivität}

Das Grundproblem bei der Quantifizierung von KA ist der Mangel anerkannter Methoden. Trost (2007) zählt über 50 verschiedene Erfassungstechniken (wie z.B. Schrittzähler oder indirekte Kaloriemetrie), wobei der Einsatz einer Methode abhängig vom jeweiligen Untersuchungsziel ist (siehe im Überblick Trost, 2007; Woll,
2004; Welk, 2002). Einer der größten Vorteile der Fragebogenmethode bei der Aktivitätserfassung liegt in ihrer Vielseitigkeit (Beneke \& Leithäuser, 2009). Neben der Erfassung von Dauer, Häufigkeit und Intensität können bei Fragebogenmethoden auch Informationen über die Art der KA aufgezeichnet werden, was bei apparativen Verfahren i.d. R. nicht möglich ist. Auch der Aspekt der Reaktivität spielt bei der Fragebogenmethode eine wesentlich geringere Rolle als bei apparativen Verfahren (z. B. Schrittzähler oder elektronische Aktivitätsmonitore). Des Weiteren wird im Rahmen epidemiologischer oder auch gesundheitswissenschaftlicher Fragestellungen zumeist auf größere Stichproben zurückgegriffen, weshalb aus praktischen und finanziellen Gründen die Fragebogenmethode oft die einzig praktikable Alternative darstellt (vgl. Matthews, 2002). Fragebogenmethoden zeigen aber auch eine Reihe von Defiziten, wie zum Beispiel das sozial erwünschte Antwortverhalten (Schwarz \& Oyserman, 2001). Außerdem ist wenig darüber bekannt, wie Personen körperliche Aktivitäten kodieren, nach welchen Dimensionen und Domänen sie die jeweilige KA differenzieren, wie sie gespeichert und anschließend wieder abgerufen werden (Woll, 2004; Fuchs, 2003; Matthews, 2002). Aus diesem Grund ist der Prüfung der Reliabilität und Validität - als eine Voraussetzung für die Anwendung der Fragebogenmethode eine umso höhere Bedeutung beizumessen.

\section{Forschungsdesiderate und Zielstellung}

Eines der größten Defizite der deutschsprachigen Aktivitätsforschung im Kindes- und Jugendalter liegt darin begründet, dass es fast genauso viele Aktivitätsfragebögen wie Untersuchungen gibt. Dies wirkt sich negativ auf die Vergleichbarkeit der Studien aus. Ein weiteres zentrales Problem ist, dass derzeit auf Reliabilität und Validität geprüfte Fragebögen für Kinder und Jugendliche in deutscher Sprache kaum existieren. Bei der Betrachtung der internationalen Literatur sind zwar einige auf Reliabilität und Validität geprüfte Fragebögen zur Erfassung von KA von Jugendlichen (siehe Tabelle 1) zu finden, diese sind aber aufgrund von kulturellen und gesellschaftlichen Unterschieden kaum auf deutsche Verhältnisse übertragbar. So z. B. ist die Vereinsstruktur, wie wir sie in Deutschland in der Regel als Nonprofit-Organisation mit ehrenamtlichen Helfern kennen, im angelsächsischen Raum kaum vorhanden. Der Sport im Rahmen von Schulmannschaften ist dagegen in den USA sehr verbreitet, in Deutschland dagegen kaum. Darüber hinaus werden im englischsprachigen Raum meistens solche Aktivitäten zum Sport (,sports') gezählt, die einen Leistungscharakter haben. Im deutschsprachigen Raum zählen auch gesundheitsorientierte Aktivitäten (z.B. ins Fitnessstudio gehen) zur Sportaktivität. 


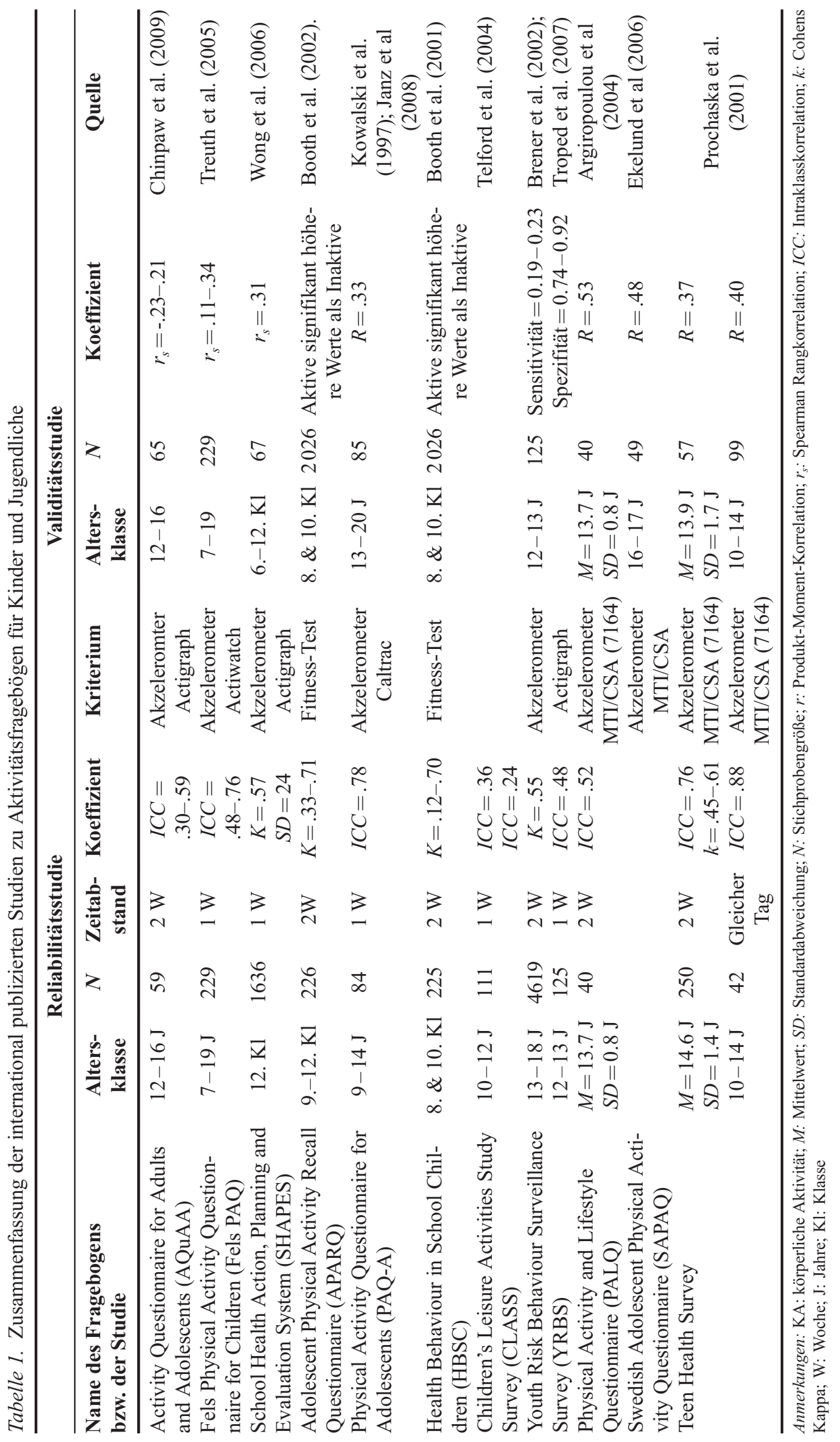


Um diese Forschungslücke zu schließen, wurde im Rahmen der MoMo-Studie ${ }^{1}$ (Motorik-Modul) ein deutschsprachiger Aktivitätsfragebogen (MoMo-AFB) zur Erfassung der habituellen KA für Kinder und Jugendliche entwickelt und eingesetzt. Ziel der vorliegenden Studie ist die Prüfung der Reliabilität und der kriterienbezogenen Validität des MoMo-AFB.

\section{Methoden}

\section{Stichprobe}

An der Untersuchung nahmen bei der ersten Erhebung 224 Schülerinnen und Schüler der Klassen fünf bis neun aller Schulformen einer Gesamtschule im Landkreis Konstanz teil. Von diesen 224 Schülerinnen und Schülern waren 196 Schülerinnen und Schüler bei der zweiten Erhebung anwesend, die damit die Stichprobe der vorliegenden Studie darstellen. Die Jugendlichen hatten ein Durchschnittsalter von $12.8 \quad(S D=1.6) \quad J a h r e n$. Die jüngsten Teilnehmerinnen und Teilnehmer waren neun, die ältesten 17 Jahre alt. Die Stichprobe setzte sich aus 109 (56\%) männlichen und 87 (44\%) weiblichen Probanden zusammen. Von den teilnehmenden Schülerinnen und Schülern besuchten 28 (14\%) die Hauptschule, 63 (32\%) die Realschule und 105 (54\%) das Gymnasium. In den fünften Klassen nahmen $58(30 \%)$, in den sechsten Klassen 14 (7\%), in den siebten Klassen 55 (28\%), in den achten Klassen 40 (20\%) und in den neunten Klassen 29 (14\%) Schülerinnen und Schüler an der Studie teil. Die Teilnahme an der Untersuchung erfolgte freiwillig. Von den 28 Drop-outs waren 15 Jungen und 13 Mädchen mit einem Durchschnittsalter von 12.2 Jahren $(S D=1.8)$. Vier Schülerinnen und Schüler besuchten die Hauptschule, neun die Realschule und fünfzehn das Gymnasium.

\section{Untersuchungsablauf}

Die Datenerhebung erfolgte von April bis Juli 2009. Zur Ermittlung der Test-Retest-Reliabilität wurde der MoMoAFB zweimal in einem Abstand von sieben Tagen ausgefüllt. Da es sich bei körperlicher Aktivität um ein instabiles Merkmal handelt (Malina, 2001), ist ein Intervall von einer Woche zwischen zwei Messungen optimal, um die Test-Retest-Reliabilität zu schätzen. Die Schülerinnen und Schüler trugen über den gesamten Untersuchungszeitraum einen Beschleunigungssensor (Actigraph GT1M). Um Informationen über die Art der KA zu erhalten und diese mit den Daten des Beschleunigungssen-

\footnotetext{
${ }^{1}$ Das Motorik-Modul wird im Rahmen des Kinder- und Jugendgesundheitssurveys (KiGGS) mit einer deutschlandweit repräsentativen Stichprobe von 4529 Personen eingesetzt und untersucht die körperliche Leistungsfähigkeit und körperliche Aktivität von Kindern und Jugendlichen in Deutschland.
}

sors zu kombinieren, führten die Schülerinnen und Schüler während des gesamten Untersuchungszeitraums das Bewegungstagebuch „Previous Day Physical Activity Recall“ (PDPAR).

\section{Charakterisierung des MoMo-Aktivitätsfragebogens}

Der MoMo-AFB umfasst insgesamt 28 Items. Die Entwicklung der Items erfolgte in Anlehnung an eine Reihe bereits bestehender Instrumente (Bös, Opper \& Woll, 2002; Prochaska, Sallis \& Long, 2001; im Überblick Bös, Worth, Opper, Oberger \& Woll, 2009, S. 62 ff.). Der MoMo-AFB wurde theoriegeleitet-deduktiv konstruiert. Ausgehend von den theoretischen Überlegungen zum Konzept der KA (siehe oben), wurden für jede Domäne Items formuliert, die alle vier Dimensionen der KA (Art, Häufigkeit, Dauer und Intensität) widerspiegeln. Das Ziel des MoMo-AFB ist eine domänenspezifische Quantifizierung der KA in Minuten pro Woche in verschiedenen Intensitätsstufen (niedrig, mittel und hoch). Der Fragebogen dient zur Erfassung der habituellen KA.

Mit den ersten beiden Items zur allgemeinen KA wird erfragt, an wie vielen der letzten sieben Tage (Item 1) bzw. einer normalen Woche (Item 2) die Kinder und Jugendlichen für mindestens 60 Minuten am Tag aktiv waren (Prochaska et al., 2001). Die Probanden haben bei beiden Items die Möglichkeit, eine Antwort zwischen null und sieben Tagen anzukreuzen. Diese Abfrage bezieht sich auf die international vereinbarten Aktivitätsstandards (z. B. 60 Min/Woche, vgl. Corbin, Pangrazi \& Le Masurier, 2004).

Mit den verbleibenden 26 Items wird die KA in den vier Domänen KA im Alltag, KA in der Schule sowie Freizeitsport innerhalb und außerhalb von Vereinen abgebildet. Für jede dieser Domänen der KA wird die Art (z. B. Welche Sportart(en) betreibst du im Verein?), Dauer (z.B. Wie lange dauert das Training (ohne Wegzeit, Umziehen und Duschen)?), Häufigkeit (z. B. Wie häufig betreibst du die jeweilige Sportart pro Woche (im Verein)?) und Intensität (z. B. Wie sehr strengst du dich bei der jeweiligen Sportart in der Regel an?) erfragt. Zur Intensitätserfassung werden drei Antwortkategorien $(1=$ ohne Schwitzen und Kurzatmigkeit/Schnaufen; $2=$ etwas Schwitzen und Kurzatmigkeit/Schnaufen; $3=$ viel Schwitzen und Kurzatmigkeit/Schnaufen) vorgegeben. Um möglichst genaue Informationen zu erhalten, werden die Fragen nach der Dauer (Min/Training) und der Häufigkeit (Tage/Woche) offen gestellt. Da nicht alle körperlichen Aktivitäten das ganze Jahr über ausgeübt werden können (z. B. Skifahren), wird mit separaten Items erfragt, in welchen Monaten (Jan.-Dez.) die jeweiligen Aktivitäten ausgeführt werden (z. B. In welchen Monaten führst du die jeweilige Sportart aus?). Damit wird eine möglichst präzise Erhebung des Aktivitätsverhaltens über das ge- 
samte Jahr im Sinne der habituellen KA gewährleistet. Für die Domäne der KA im Alltag werden drei Aktivitäten vorgegeben: „Zufußgehen“, „Radfahren“ und „,im Freien Spielen“.

Für jede Aktivitätsdomäne wurde ein Minutenindex gebildet, indem für jede Aktivitätsart die Häufigkeit mit der Dauer der Übungseinheit multipliziert wird. Dabei wurden körperliche Aktivitäten mit mittlerer und hoher Intensität (moderate to vigorous physical activity, MVPA) berücksichtigt. In den Domänen Sport innerhalb und auBerhalb des Vereins sowie Sport in der Schule wurde eine Gewichtung mit dem Faktor Anzahl der ausgeübten Monate/12 vorgenommen, wodurch saisonale Abhängigkeiten von Sportarten wie z. B. beim Skifahren berücksichtigt werden können. Der Gesamtindex wurde aus der Summe der einzelnen Domänen gebildet. Damit lassen sich die so ermittelten Werte mit anderen Messinstrumenten zur Erfassung der körperlichen Aktivität (z. B. Akzelerometer) vergleichen und hinsichtlich der geltenden Aktivitätsrichtlinien überprüfen.

Für die vollständige Darstellung des MoMo-AFB sei auf Bös et al. (2009) verwiesen.

\section{Messinstrumente}

Akzelometer Actigraph GT1M. Um die subjektiven Daten des MoMo-AFB zu validieren, wurde der Beschleunigungssensor Actigraph GT1M verwendet. Das Gerät misst Beschleunigungen der vertikalen und horizontalen Achse. KA wird in Aktivitätseinheiten beschrieben, die in Zeitintervallen (Epochen) gespeichert werden. Eine Einheit entspricht 16.6 Milli G/Sekunde ${ }^{2}$. In der vorliegenden Untersuchung wurde eine Epochenlänge von zehn Sekunden festgelegt. Die Kinder und Jugendlichen trugen das 27 Gramm schwere Gerät mit einem elastischen Gurt an der rechten Hüfte. Akzelerometer messen die aktuelle KA objektiv, sind auch bei größeren Stichproben anwendbar (vgl. Welk, 2002) und wurden umfassend auf Reliabilität und Validität überprüft (vgl. z. B. Freedson, Pober \& Janz, 2005). Die Validität des Geräts konnte in mehreren Studien anhand „harter" Kriterien wie indirekter Kalorimetrie $(r=.86$; Trost et al., 1998) und doppelt markiertem Wasser ${ }^{3}$ $(r=.67$; Ekelund, Yngve, Brage, Wetserterp \& Sjo-

${ }^{2}$ Milli G Milli Gauß ist die Einheit der magnetischen Flussdichte im elektromagnetischen CGS-System (engl. Centimeter-Gram-SecondSystem). In der Accelerometrie wird diese Einheit verwendet, um die Beschleunigung des Körpers zu schätzen.

${ }^{3}$ Doppelt markiertes Wasser (engl. doubly labelled water) ist eine Methode zur Bestimmung des Energieverbrauchs, die sich der stabilen Isotope ${ }^{18} \mathrm{O}$ und ${ }^{2} \mathrm{H}$ bedient. Mit dieser Methode wird die Kohlendioxidproduktion aus der unterschiedlichen Abnahmegeschwindigkeit der stabilen Isotope ${ }^{18} \mathrm{O}$ und ${ }^{2} \mathrm{H}$ im Blut errechnet. Kohlendioxid wird bei der Energieumsetzung durch die Atmung frei und kann somit als Maß für den Energieverbrauch (ausgedrückt in Watt) benutzt werden. strom, 2004) bestätigt werden. Weitere Studien (z.B. Freedson et al., 2005) bestätigen die Validität des Actigraph GT1M im Hinblick auf die Bestimmung des Umfangs der KA.

Die Daten wurden in Abgleich mit dem Bewegungstagebuch hinsichtlich der verschiedenen Domänen der KA kategorisiert. Wir schätzten die Tragezeit mit einem Algorithmus in Anlehnung an Troiano et al. (2008), bei dem die Zeitschwelle auf 60 Minuten und die Activity-CountSchwelle auf 50 Activity-Counts pro Minute festgelegt wurde. Ein Messtag wurde zur Validitätsbeurteilung herangezogen, wenn das Gerät mehr als zehn Stunden pro Tag getragen wurde. Alle in die Analyse aufgenommenen Fälle mussten mindestens fünf valide Messtage aufweisen. Auf diese Weise konnten von 139 Probanden gültige Datensätze gewonnen werden.

Bewegungstagebuch. Zur Erfassung der KA auf der Tagesebene wurde der speziell für Kinder und Jugendliche entwickelte „Previous Day Physical Activity Recall“ (PDPAR) verwendet (Weston, Petrosa \& Pate, 1997). Bei diesem Instrument werden in 39 Kategorien jeweils typische Aktivitäten von Kindern und Jugendlichen (z.B. Essen, Schlafen, Schule, Freizeit, Sport) vorgegeben. Ein Werktag ist dabei von 13.00 bis $24.00 \mathrm{Uhr}$ in elf Blöcke und ein Wochenendtag von 9.00 bis $24.00 \mathrm{Uhr}$ in fünfzehn Blöcke zu je einer Stunde aufgeteilt. Zu jeder KA kreuzen die Probanden nebenstehend das Ausmaß der Intensität (gar nicht, ein wenig, mittel, sehr anstrengend) an, deren Abstufung für körperliche Aktivitäten mit substantiellen Energieverbrauch im Wesentlichen der Intensitätsabstufung des MoMo-AFB entspricht. Für die Werktage wird darüber hinaus erfragt, ob und in welcher Stunde an dem Tag Sportunterricht oder sonstige sportliche Aktivitäten in der Schule stattgefunden haben. Damit sollte eine zeitgenaue Quantifizierung der sportlichen Aktivität in der Schule gewährleistet werden. Der morgendliche Weg zur Schule (z. B. per Fahrrad) wird jedoch nicht berücksichtigt. Der PDPAR hat sich in mehreren Studien (z. B. Pate et al., 2003, 2005; Trost, Ward, McGraw \& Pate, 1999; Weston et al., 1997) als zufriedenstellend reliabel und valide erwiesen.

\section{Auswertungsstrategien und Indexbildung}

Da es sich beim MoMo-AFB um ein formatives Messmodell handelt, werden die Reliabilität des Fragebogens mit Hilfe der Test-Retest-Reliabilität und die Validität anhand der kriterienbezogenen Validität geschätzt. Eine Untersuchung der internen Struktur anhand der Faktorenanalyse sowie der internen Konsistenz ist kein adäquates Verfahren für ein formatives Messmodell (siehe Bagozzi, 1994, S. 333) und wird deshalb nicht angewendet. 
Um die Übereinstimmung der Angaben seitens der Schülerinnen und Schüler zu beiden Messzeitpunkten auf der Itemebene zu ermitteln (Test-Retest-Reliabilität), wurden die Cohens Kappa-Koeffizienten für kategoriale Antwortformate und gewichtete Kappakoeffizienten für ordinal- und intervallskalierte Items berechnet. Die Interpretation der gewichteten und ungewichteten KappaKoeffizienten basiert auf dem Klassifikationssystem von Landis und Koch (1977). Hiernach indiziert ein KappaKoeffizient $<.10$ schlechte, $.10-.20$ geringe, $.21-.40$ ausreichende, $.41-.60$ mittlere, $.61-.80$ gute und $.81-1.00$ sehr gute Übereinstimmung.

Zur Schätzung der Test-Retest-Reliabilität auf der Ebene der Indizes wurden Intraklassenkorrelationen $\left(I C C_{2,1}\right)$ anhand des two-way random effects Modells berechnet, in dem sowohl Subjekte als auch die Zeit zufällig sind (Shrout \& Fleiss, 1979). Dieses Modell schätzt die Übereinstimmung (agreement) der Angaben nach einer Woche.

Der Anteil der fehlenden Daten für den MoMo-AFB war sehr gering und bewegte sich zwischen $0 \%$ und $2 \%$. Das Bewegungstagebuch wurde von 189 Probanden bearbeitet und vom Actigraph GT1M lagen die Daten von 139 Personen vor.

Um die Übereinstimmungen zwischen dem MoMoAFB (in MVPA-Minuten pro Woche) und dem Actigraph GT1M (in MVPA-Minuten pro Woche) festzustellen, werden Spearman-Rangkorrelationen berechnet, da bei den Akzelerometerdaten meist eine linksschiefe Verteilung vorlag. Dabei wird der MoMo-AFB sowohl als Gesamtindex als auch domänenspezifisch (Schulsport, Alltagsaktivitäten, Freizeitsport außerhalb und innerhalb des Vereins) auf die kriterienbezogene Validität untersucht. Verwendet wurden die Fragebogendaten des ersten Messzeitpunktes.

\section{Ergebnisse}

\section{Deskriptive Statistiken}

In Tabelle 2 sind Mittelwerte und Standardabweichungen für die Variable „Alter“" sowie die deskriptiven Werte der KA der drei Messinstrumente zur Erfassung der KA dargestellt. Die Ergebnisse verdeutlichen, dass Jungen bezüglich aller drei Messinstrumente zur Erfassung der KA höhere Werte aufweisen als Mädchen. In der Gegenüberstellung der Altersgruppen zeigen sich entsprechend höhere Werte zugunsten der jüngeren Schülerinnen und Schüler (9-13-Jährige). Die anhand des Akzelerometers geschätzten Mittelwerte für die Aktivitätsminuten in MVPA liegen deutlich niedriger als die Mittelwerte des MoMo-AFB und des PDPAR.

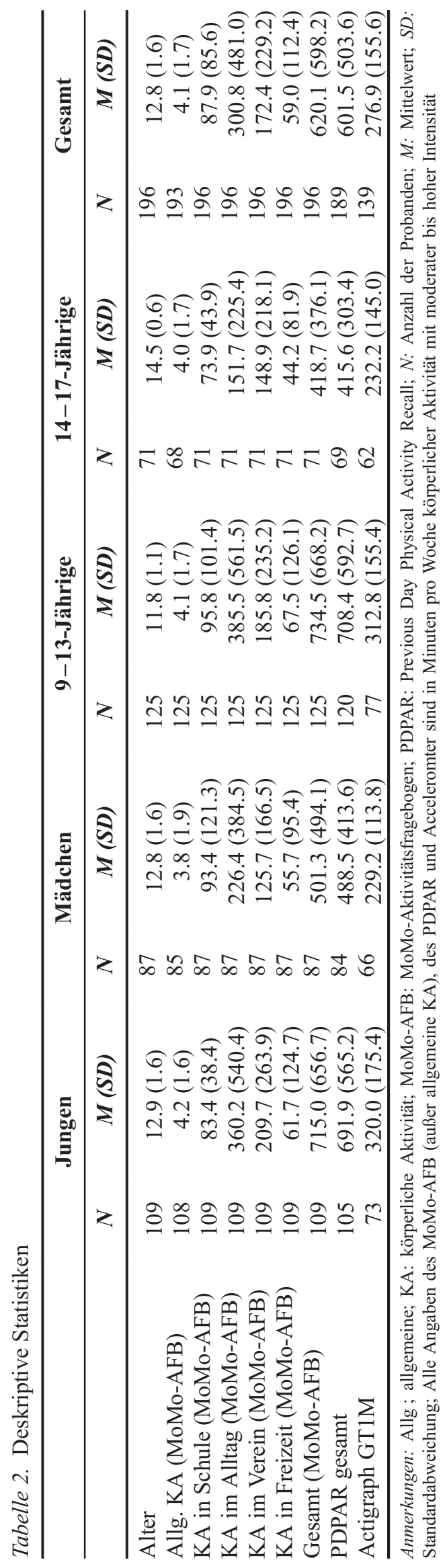




\section{Test-Retest-Reliabilität}

In Tabelle 3 sind die Koeffizienten zur Schätzung der TestRetest-Reliabilität differenziert nach den Domänen angegeben. Die Test-Retest-Reliabilität wurde sowohl auf der Item- als auch auf der Indexebene geprüft. Auf der Itemebene zeigt sich eine Spannweite von niedriger bis zu sehr guter Reliabilität, wobei die Items zur allgemeinen KA mit einem durchschnittlichen $k=.54$ die geringste Test-Restest-Reliabilität aufweisen, während die Übereinstimmung für den Bereich des Freizeitsports in Vereinen mit einem durchschnittlichen Kappa-Koeffizienten von $k=.81$ am größten sind. Über alle berücksichtigten Items betrachtet, beträgt der durchschnittliche KappaKoeffizient $.66(S D=.19)$. Insgesamt bewegen sich die Kappa-Koeffizienten von 8 Items (29\%) im Bereich zwischen .81 und 1.00, von 7 Items (25\%) im Bereich von .61 und .80, von weiteren 11 Items (39\%) im Bereich von .41 und .60 und von den restlichen 2 Items $(7 \%)$ im Bereich von .21 und .40 .

Betrachtet man die Test-Retest-Korrelation auf der Indexebene, so ergibt sich für den Gesamtindex eine Intraklassenkorrelation (ICC) von $r=.68$. Die höchste Intraklassenkorrelation ist für die Domäne der allgemeinen KA mit $r=.74$ festzustellen. Bei der Betrachtung der ICC in verschiedenen Domänen in Abhängigkeit von Alter und Geschlecht ist zu erkennen, dass ältere Teilnehmerinnen und Teilnehmer (14-17-Jährige) höhere ICC-Werte aufweisen.

\section{Validität}

Zur Beurteilung der kriterienbezogenen Validität wurden die Rangkorrelationen nach Spearman zwischen den MVPA-Minutenindizes des MoMo-AFB und den mittels Actigraph GT1M gemessenen Werten berechnet (siehe Tabelle 4). Der Gesamtindex des MoMo-AFB korreliert mit der Summe der Angaben des Akzelerometers der entsprechenden Woche mit einem Korrelationskoeffizienten von $r=.29$ hoch signifikant. Betrachtet man die Korrelationen in unterschiedlichen Domänen, so ergeben sich deutliche Unterschiede. Nicht signifikante Korrelationen zeigen sich in den Domänen KA in der Schule $(r=.04)$, KA im Alltag $(r=.09)$ und Freizeitsport außerhalb der Vereine mit $r=.10$. Die höchsten Korrelationen sind für den Index Freizeitsport in Vereinen und beim Gesamtindex mit $r=.35$ bzw. $r=.29$ zu finden.

\section{Diskussion}

Das Ziel der vorliegenden Untersuchung ist die Überprüfung der Test-Retest-Reliabilität und der kriterienbezogenen Validität des MoMo-AFB. 


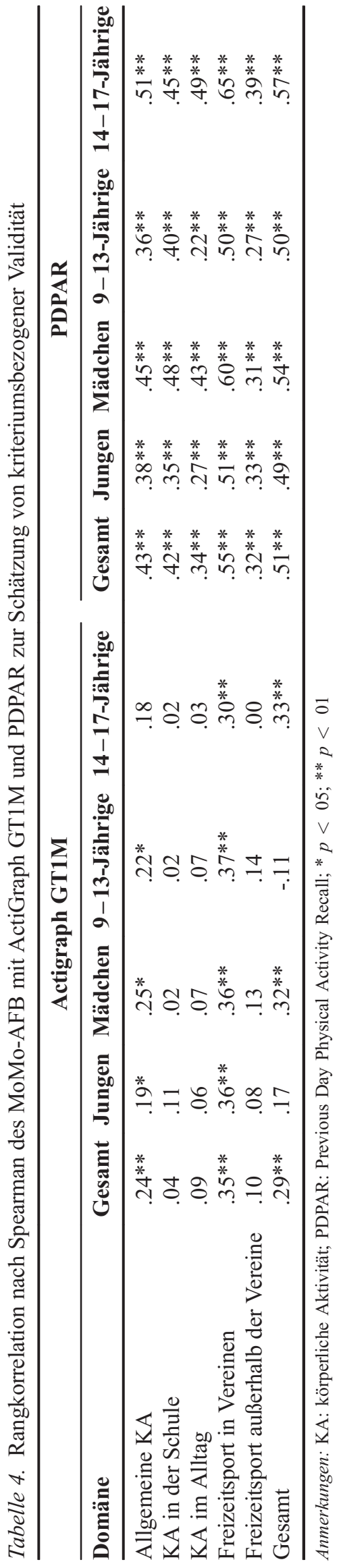

\section{Mittelwertsvergleiche}

Die Ergebnisse der Mittelwertsvergleiche zwischen MoMoAFB und den beiden Validierungsinstrumenten liefern ein differenziertes Bild. Übereinstimmend mit den beiden Instrumenten zur Erfassung der aktuellen KA zeigt sich beim MoMo-AFB, dass Jungen körperlich aktiver sind als Mädchen und dass jüngere Schüler aktiver sind als ältere Schüler. Bezüglich der Mittelwertvergleiche zwischen den Messinstrumenten zeigt sich, dass die mittels MoMo-AFB und PDPAR gewonnenen Daten deutlich höhere Mittelwertsschätzungen liefern als die Daten, die anhand des Actigraph GT1M erhoben wurden. Dieses Ergebnis spiegelt den Unterschied zwischen subjektiven und objektiven Messverfahren wider. Während die Mittelwerte von MoMo-AFB und PDPAR sehr eng beieinander liegen, sind die Mittelwertschätzungen anhand des Actigraph GT1M nur etwa halb so hoch. Diese Ergebnisse decken sich mit den Erkenntnissen von Wong, Leatherdale und Manske (2006), die anhand des SHAPES-Aktivitätsfragebogens deutlich höhere Mittelwerte ermittelten als anhand des Akzelerometers. Ein Ansatz zur Erklärung der Mittelwertsunterschiede ist darin zu sehen, dass die Kinder und Jugendlichen anhand ihrer subjektiven Angaben die tatsächliche MVPA überschätzen. Es ist anzunehmen, dass die Jugendlichen nicht in der Lage sind, die Informationen im Gedächtnis korrekt zu kodieren, zu speichern und abzurufen oder auch zu sozial erwünschtem Antwortverhalten neigen (Schwarz \& Oyserman, 2001). Andererseits ist es auch möglich, dass die mit Hilfe des Akzelerometers erfasste MVPA unterschätzt wird, da einzelne Aktivitäten nicht berücksichtigt (z.B. Schwimmen) bzw. unterschätzt werden (z.B. Radfahren). Unserer Einschätzung nach ist es naheliegend, dass beide Erklärungen zutreffen.

\section{Reliabilität}

Die Reliabilität des MoMo-AFB wurde anhand eines Vergleichs zwischen zwei Erhebungen mit einem Abstand von sieben Tagen geprüft. Die Test-Retest-Reliabilität kann auf der Itemebene als zufriedenstellend bewertet werden. Nach Maßstäben von Landis und Koch (1977) zeigen 54 \% der Items eine gute bis sehr gute und weitere $39 \%$ eine mittlere Übereinstimmung. Lediglich zwei Items, die die Intensität der KA in der Schule und im Alltag erfassen, weisen eine geringe Übereinstimmung auf. Dieser Befund deutet im Allgemeinen darauf hin, dass den Jugendlichen die Abschätzung der Intensität von KA besonders schwer fällt. Da sich die Intensität der KA im Laufe der Stunde bzw. der Trainingseinheit ständig ändert, ist eine Abschätzung der Intensität für die gesamte Stunde bzw. Trainingseinheit besonders schwierig. Der durchschnittliche Kappa-Koeffizient beträgt .66 und ist vergleichbar mit den Ergebnissen zum SHAPES-Aktivitätsfragebogen (School Health Action, Planning and Evaluation System; Wong et al., 2006), der ebenfalls habituelle KA erfasst und in Bezug auf Alter und Geschlecht mit einer vergleichbaren Stichprobe überprüft 
wurde. Etwas niedriger liegen die Kappa-Koeffizienten für den Aktivitätsfragebogen der HBSC-Studie (Health Behaviour in School Children; Booth, Okely, Chey \& Bauman, 2001), den Aktivitätsfragebogen der YBRS-Studie (Youth Risk Behaviour Surveillance System; Brener et al., 2002) und den Adolescent Physical Activity Recall Questionnaire (APARQ; Booth, Okely, Chey \& Bauman, 2002), bei deren Untersuchung jedoch ein Abstand von zwei Wochen zwischen den Messungen gewählt wurde, was die vergleichsweise geringen Kappa-Koeffizienten erklären könnte.

Eine weiterführende Interpretation der Kappa-Koeffizienten legt nahe, dass der Freizeitsport im Verein und die KA in der Schule die höchsten absoluten Übereinstimmungen aufweisen. Ein Grund dafür könnte sein, dass $\mathrm{KA}$ in Vereinen und in der Schule mit einer festgelegten Anzahl von Sportstunden strukturierter abläuft und sich über die Zeit vergleichsweise stabiler zeigt, was die Erinner- und Abrufbarkeit dieser Gedächtnisinformationen erhöht. Passend zu diesen Überlegungen ist die Tatsache, dass allgemeine KA und Alltagsaktivitäten die niedrigsten Übereinstimmungskoeffizienten aufweisen. Gerade die Alltagsaktivitäten sind in der Regel eher spontan und weisen niedrige Stabilität auf. Allgemeine KA stellt ein Konglomerat von körperlichen Aktivitäten in verschiedenen Domänen dar. Schwankungen in einer Domäne können absolute Veränderungen in diesem ganzheitlichen Aspekt der KA hervorrufen.

Betrachtet man die Reliabilität auf der Indexebene (in Minuten pro Woche), beträgt die Intraklassenkorrelation (ICC) für den Gesamtindex .68. Dieses Ergebnis ist ebenfalls vergleichbar mit den $I C C$-Koeffizienten anderer internationaler Aktivitätsfragebögen. So ermittelten Treuth, Hou, Young \& Mayland (2005) für den Gesamtindex des Fels-PAQ (Fels Physical Activity Questionnaire for Children), der anhand einer vergleichbaren Stichprobe und mit gleichem zeitlichen Abstand zwischen den Messungen auf Reliabilität geprüft wurde, einen $I C C$-Koeffizient von .67. Etwas niedrigere ICC-Koeffizienten konnten für den APARQ (Booth et al., 2002), AQuAA (Activity Questionnaire for Adults and Adolescents; Chinapaw, Slootmaker, Schuit, van Zuidam \& van Mechelen, 2009), CLASS-Aktivitätsfragebogen (Children's Leisure Activities Study Survey; Telford, Salmon, Jolley \& Crawford, 2004), PALQ (Physical Activity and Exercise Questionnaire; Argiropoulou Michalopoulou, Aggeloussis \& Avgerinos, 2004) und YRBS-Aktivitätsfragebogen (Troped et al., 2007) festgestellt werden. Dagegen zeigten sich für den PAQ-A (Physical Activity Questionnaire for Adolescents; Janz, Letuchy, Wenthe \& Levy, 2008) sowie für den Fragebogen der Teen Health Study (Prochaska et al., 2001) etwas höhere Intraklassenkorrelationen.

Bei einer domänenspezifischen Betrachtung weist die nach Prochaska et al. (2001) erfasste allgemeine KA den höchsten ICC von .74 auf. Interessant ist dabei festzustellen, dass die allgemeine KA auf der Itemebene die niedrigsten Test-Retest-Koeffizienten aufweist. Dieses Ergebnis impliziert, dass dieses globale Maß eine relativ niedrige absolute Übereinstimmung im Sinne des Kappakoeffizienten zeigt, wobei die durch die $I C C$ eher repräsentierte normative Stabilität vergleichsweise hoch ist. Diese Befunde entsprechen im Wesentlichen den Befunden von Prochaska et al. (2001), die einen ICC-Koeffizieten von .76 für einen Zeitraum von zwei Wochen fanden. Die Cohens Kappa-Koeffizienten bewegten sich zwischen .45 und .61 .

Eher unerwartet ist der Befund, dass der Index für KA in der Schule die niedrigsten $I C C$-Koeffizienten zeigt. Gerade die Quantifizierung der KA in der Schule müsste verhältnismäßig leicht fallen, da die Aktivitätseinheiten wie Schulsport und Sport-AG in einem feststehenden Rahmen stattfinden. Für den Index KA im Alltag fällt der ICC mit .61 ebenfalls niedrig aus. Dieser Befund deckt sich mit den Ergebnissen von Treuth et al. (2005) und Booth et al. (2002), wo für Alltags- und nichtorganisierte körperliche Aktivitäten niedrigere $I C C$-Koeffizienten zu finden sind. Wie oben erwähnt könnte eine mögliche Erklärung für die niedrigen Koeffizienten sein, dass nichtstrukturierte Alltagsaktivitäten im Vergleich zu strukturierten körperlichen Aktivitäten (z. B. Vereinsport) mit wöchentlich feststehenden Terminen aus der Erinnerung schwieriger zu berichten und weniger stabil sind (vgl. Booth et al., 2002).

Abweichend von der Fels- und APARQ-Studie ist in unserer Studie die ICC für Jungen mit .71 höher als für Mädchen mit .62. Während Treuth et al. (2005) für den Fels-Aktivitätsfragebogen keine Geschlechterunterschiede feststellen, sind die ICC-Koeffizienten für den APARQ bei Mädchen höher als bei Jungen (Booth et al., 2002). Da diese Korrelationsunterschiede jedoch eher gering sind, könnten sie auch durch die Eigenheiten der jeweiligen Stichprobe zustande kommen. Nur in der Domäne des Vereinssports weisen Mädchen sowohl auf der Item- als auch auf der Indexebene etwas höhere Reliabilitätskoeffizienten auf als Jungen.

In Analogie zum Fels-PAQ sind die für den MoMoAFB ermittelten altersspezifischen Unterschiede eher gering einzuschätzen. Sowohl bei einer Betrachtung auf der Item- als auch auf der Indexebene sind die altersspezifischen Unterschiede nur bei den Domänen der allgemeinen KA, der KA in der Schule und dem Vereinssport festzustellen. Die älteren Jugendlichen (14-17-Jährigen) weisen dabei höhere Test-Retest-Korrelationen auf als die jüngeren Teilnehmer (9-13-Jährigen). Booth et al. (2002) finden dagegen zum Teil relativ große $I C C$-Unterschiede zwischen der achten und der zehnten Klasse.

\section{Validität}

Die Validität des MoMo-AFB wurde anhand des Akzelerometers Actigraph GT1M und des Bewegungstagebuchs PDPAR geprüft. 
Akzelerometer. Der Gesamtindex des MoMo-AFB weist eine signifikante Rangkorrelation nach Spearman von .29 mit den Akzelerometerdaten auf. Auch wenn die signifikante Korrelation eher niedrig ausfiel, schneidet der MoMo-AFB in dieser Studie besser ab, als in einer Studie mit insgesamt 73 Jugendlichen bei der für den Gesamtindex eine Korrelation von $r=.11$ festgestellt wurde (Kahlert \& Brand, 2011). Darüber hinaus sind die vorliegenden Ergebnisse vergleichbar mit anderen Studien, bei denen Akzelerometer zur Validierung der Aktivitätsfragebögen für Kinder und Jugendliche eingesetzt wurden. Vergleichbare Rangkorrelationen des Gesamtindexes mit den Akzelerometerdaten wurden für den SHAPESAktivitätsfragebogen mit $r=.31$ (Wong et al., 2006), für den Fels-PAQ mit $r$ zwischen .34 und .11 (Treuth et al., 2005) für den Swedish Adolescent Physical Activity Questionnaire (SAPAQ; Ekelund, Neovius, Linne \& Rössner, 2006) mit $r=.48$ und für den Physical Activity Questionnaire for Adolescents (PAQ-A; Kowalski, Crocker \& Kowalski, 1997) mit $r=.33$ festgestellt. Dagegen waren in einer Stichprobe mit 111 Kindern und Jugendlichen im Alter zwischen zehn und zwölf Jahren die Rangkorrelationen zwischen dem Aktivitätsfragebogen der CLASS-Studie (Children's Leisure Activities Study Survey) und den Akzelerometerdaten von Actigraph MTI nicht signifikant (Telford et al., 2004), was weitgehend mit unseren Befunden korrespondiert. In der vorliegenden Studie wurde für die jüngere Alterklasse (9-13-Jährige) ebenfalls eine nicht signifikante Rangkorrelation gefunden. Insgesamt sind geschlechts- und altersspezifische Unterschiede festzustellen. Generell werden bei den Angaben von Mädchen sowie älteren Schülerinnen und Schülern höhere Rangkorrelationen festgestellt.

Bei einer domänenspezifischen Betrachtung sind signifikante Validitätskoeffizienten lediglich in den Domänen Freizeitsport in den Vereinen mit $r=.35$ und allgemeine KA mit $r=.24 \mathrm{zu}$ erkennen. Eine solche Tendenz wird auch in der Studie von Treuth et al. (2005) deutlich, in der die höchsten Rangkorrelationen für den Sportindex zu finden sind. In der vorliegenden Studie liegen die höchsten Rangkorrelationen in der Domäne des Vereinssports für beide Geschlechter und Altersgruppen vor. Dieses Ergebnis könnte dadurch zustande kommen, dass sportliche Aktivitäten im Verein reliabler erfasst werden und dadurch auch valider sein können. Diese Tendenz zeigt sich unabhängig von Geschlecht und Alter.

Bewegungstagebuch. Insgesamt korrelieren die anhand des PDPAR erfassten Aktivitätsdaten sowohl in den Gesamtindizes als auch in den Domänen signifikant mit dem MoMo-AFB. Die Korrelation zwischen den Gesamtindizes der beiden Maße beträgt .51 und bewegt sich in einem moderaten Bereich. Die Unterschiede zwischen Jungen und Mädchen scheinen dabei eher gering zu sein. Bei einem Vergleich zwischen den Altersgruppen ist konsistent über alle Domänen festzustellen, dass ältere Schüler höhere Korrelationen zeigen als jüngere Schüler. Damit scheint das
Alter eine wichtige Moderatorvariable bei der Beurteilung der Validität des MoMo-AFB zu sein. Ältere Schüler sind nach den vorliegenden Ergebnissen besser in der Lage ihr Aktivitätsverhalten einzustufen als jüngere Schüler.

In der domänenspezifischen Betrachtung werden die höchsten Korrelationen für den Index „Sportliche Aktivität im Verein“" ermittelt. Auch hier könnte die Erklärung herangezogen werden, dass sich die hohe Reliabilität der Domäne positiv auf ihre Validität auswirkt. Die niedrigsten Korrelationen sind in den Domänen „KA im Alltag“ und „Freizeitsport außerhalb von Sportvereinen“ zu verzeichnen, die wiederum von den verhältnismäßig niedrigen Reliabilitäten negativ beeinflusst sein könnten.

\section{Diskussion der Methoden}

Es existieren verschiedene Methoden zur Erfassung der KA von Kindern und Jugendlichen, die sich hinsichtlich des Informationstyps (subjektive vs. objektive Methoden) und des Aggregationszeitraums (aktuelle vs. habituelle Maße der KA) unterscheiden lassen. Im Rahmen dieser Studie wurden drei Messinstrumente verwendet: ein subjektives Maß der habituellen KA (MoMo-AFB), ein subjektives Maß der aktuellen KA (PDPAR) und ein objektives Maß der aktuellen KA (Actigraph GT1M). Wie in einer Vielzahl vorausgegangener empirischer Studien festgestellt (vgl. z. B. Hagstromer, Ainsworth, Oja \& Sjostrom, 2010) liefern die subjektiven Maße der KA auch im Rahmen dieser Studie wesentlich höhere Schätzungen der Mittelwerte der KA als das objektive Maß. Inwieweit es sich hier um Überschätzungen der KA anhand der subjektiven Maße bzw. Unterschätzungen anhand des objektiven Maßes handelt, lässt sich nicht abschließend beurteilen. Auf der einen Seite gibt es Gründe anzunehmen, dass im Falle der Fragebogenmethode - bei Enkodierung und Erinnerung der Information aus dem Gedächtnis Verzerrungen auftreten, die eine Überschätzung der Dauer und Intensität zur Folge haben (Watkinson et al., 2010). Gerade die unstrukturierten und unregelmäßigen Aktivitäten im Alltag und im Freizeitsport lassen sich schwierig aus dem Gedächtnis korrekt abrufen. Deshalb stellt sich die Frage, inwieweit die Jugendlichen die für die Bearbeitung des MoMo-AFB notwendigen kognitiven Anforderungen erfüllen können. Auf der anderen Seite ist zu vermuten, dass der Akzelerometer das Ausmaß der KA bei bestimmten Typen der KA unterschätzt (z. B. bei Bewegungen der Arme, Bergaufgehen, Heben von Gewichten, Radfahren oder Tragen von Objekten) bzw. gar nicht erfasst (z. B. Schwimmen, vgl. Crouter, Churilla \& Bassett, 2006). Wir gehen daher davon aus, dass die Akzelerometerdaten zur Unterschätzung des Ausmaßes der KA führen, wohingegen es anhand von subjektiven Maßen eher zu einer Überschätzung kommen dürfte. Eine Kombination der subjektiven und objektiven Methoden könnte deshalb eine Methode der Wahl sein, da die Vorteile der beiden 
Methodenarten genutzt und die Schwachstellen weitgehend ausgeschaltet werden können.

\section{Limitationen}

In der Interpretation der vorliegenden Befunde sind nachfolgende Limitationen $\mathrm{zu}$ berücksichtigen: Erstens gründen die alters- und geschlechtsspezifischen Analysen auf verhältnismäßig kleinen Substichproben. Zweitens ist zu bedenken, dass die vorliegenden Daten anhand einer selektiven Stichprobe gewonnen wurden. Untersucht wurden baden-württembergische Schülerinnen und Schüler, die vorwiegend in einer städtischen Region wohnen. Drittens besuchten alle Schülerinnen und Schüler zum Zeitpunkt der Untersuchung eine Gesamtschule. Inwieweit diese Ergebnisse auf andere Bundesländer bzw. Schulsysteme generalisiert werden können, muss in weiteren Studien geklärt werden. Viertens sind die verwendeten Validierungsinstrumente (der Akzelerometer und das Bewegungstagebuch) selbst nur eingeschränkt valide, was eine Bewertung der Validität des MoMo-AFB erschwert. Außerdem bleibt mit der vorliegenden Studie unbeantwortet, inwiefern sich der MoMo-AFB für Kinder und Jugendliche unterschiedlicher Bildungsniveaus (Schulformen) eignet, da aufgrund der kleinen Subgruppen in der vorliegenden Untersuchung keine Differenzierung nach der Schulform möglich war.

\section{Fazit}

Die Ergebnisse dieser Studie zeigen, dass der MoMo-AFB im Vergleich zu Akzelerometerdaten höhere Schätzungen der KA liefert, was zum Teil auf die Überschätzungen der selbsteingeschätzten KA hindeutet. Die Reliabilität und Validität des Messinstruments sind eingeschränkt und erweisen sich zum Teil abhängig von Alter und Geschlecht. Ferner zeigen die Ergebnisse, dass KA im Verein deutlich reliabler und valider erfasst wird als in anderen Domänen der KA. Sowohl die Reliabilitäts- als auch die Validitätsdaten sind jedoch vergleichbar mit den Daten anderer international verwendeter Aktivitätsfragebögen für die gleiche Altersgruppe. Unter dem Vorbehalt der Notwendigkeit weiterer Reliabilitäts- und Validierungsstudien, die vor allem auch auf eine Optimierung der bislang mittelmäßig reliablen und wenig validen Indizes zielen sollten, stellt der MoMo-AFB ein mit anderen (internationalen) Aktivitätsfragebögen für Jugendliche vergleichbares Instrument zur Erfassung der KA von Jugendlichen dar.

\section{Literatur}

Argiropoulou, E. C., Michalopoulou, M., Aggeloussis, N. \& Avgerinos, A. (2004). Validity and reliability of physical activity measures in Greek high school age children. Journal of Sports Science and Medicine, 3, 147159.
Bagozzi, R.P. (1994). Structural Equation Models in Marketing Research: Basic Principles. In R. P. Bagozzi (Ed.), Principles in Marketing Research (pp. 317 385). Cambridge: Wiley.

Becker, P., Bös, K., Opper, E., Woll, A. \& Wustmans, A. (1996). Vergleich von Hochgesunden, Normal und Mindergesun den in gesundheitsrelevanten Variablen. Zeitschrift für Ge sundheitspsychologie, 4, 5576.

Beneke, R. \& Leithäuser, R. M. (2009). Körperliche Aktivität im Kindesalter Messverfahren. Deutsche Zeitschrift für Sportmedizin, 59, 215222.

Booth, M. L., Okely, A. D., Chey, T. \& Bauman, A. (2001). The reliability and validity of the physical activity questions in the WHO health behaviour in schoolchildren (HBSC) sur vey: A population study. British Journal of Sports Medicine, 35, 263267.

Booth, M. L., Okely, A. D., Chey, T. \& Bauman, A. (2002). The reliability and validity of the Adolescent Physical Activity Recall Questionnaire. Medicine and Science in Sports and Exercise, 34, 19861995.

Bös, K., Opper, E. \& Woll, A. (2002). Fitness in der Grund schule: Förderung von körperlich sportlicher Aktivität, Haltung und Fitness zum Zwecke der Gesundheitsförderung und Unfallverhütung: Endbericht. Wiesbaden: BAG

Bös, K., Worth, A., Opper, E., Oberger, J. \& Woll, A. (2009). Motorik Modul: Eine Studie zur motorischen Leistungsfä higkeit und körperlich sportlichen Aktivität von Kindern und Jugendlichen in Deutschland. Baden Baden: Nomos.

Brener, N., Kann, L., McManus, T., Kitchen, S., Sundberg, E. \& Ross, J. (2002). Reliability of the 1999 Youth Risk Hehavior Survey Questionnaire. Journal of Adolescent Health, 31, 336342.

Caspersen C. J., Powell K. E. \& Christensen G. M. (1985). Physical activity, exercise and physical fitness: definitions and distinctions for health related research. Public Health Reports, 100, 126131.

Chinapaw, M. J. M., Slootmaker, S. M., Schuit, A. J., van Zui dam, M. \& van Mechelen, W. (2009). Reliability and Validity of Activity Questionnaire for Adults and Adolescents (AQuAA). BMC Medical Research Methodology, 9, 58.

Corbin, C. B., Pangrazi R. P. \& Le Masurier G. C. (2004). Physical activity for children: current patterns and guide lines. Research Digest, 5,1 8.

Crouter S. E., Churilla J. R. \& Bassett D. R. Jr. (2006). Estima ting energy expenditure using accelerometers. European Journal of Applied Physiology, 98, 601612.

Dishman, R. K., Washburn, R. A. \& Heath, G. W. (2004). Physical activity epidemiology. Champaign, IL: Human Kinetics.

Ekelund, U., Yngve, A., Brage, S., Westerterp, K. \& Sjostrom, M. (2004). Body movement and physical activity energy expenditure in children and adolescents: how to adjust for differences in body size and age. The American Journal of Clinical Nutrition, 79, 851856.

Ekelund, U., Neovius, M., Linne, Y. \& Rössner, S. (2006) The criterion validity of a last 7 day physical activity ques tionnaire (SAPAQ) for use in adolescents with a wide va riation in body fat: The Stockholm Weight Development Study. International Journal of Obesity, 39, 10191021.

Freedson, P., Pober, D. \& Janz, K. F. (2005). Calibration of accelerometer output for children. Medicine and Science in Sports Exercise, 37 (Suppl.), 523530.

Fuchs, R. (2003). Sport, Gesundheit und Public Health. Göt tingen: Hogrefe. 
Hagstromer, M., Ainsworth, B. E., Oja, P. \& Sjostrom, M. (2010). Comparison of subjective and an objective measure of physical activity in a population sample. Journal of Physical Activity and Health, 7, 541550.

Janz, K. F., Letuchy, E. M., Wenthe, P. \& Levy, S. M. (2008). Measuring activity in children and adolescents using self report: PAQ C and PAQ A. Medicine and Science in Sports and Exercise, 40, 767772.

Kahlert, D. \& Brand, R. (2011). Befragungsdaten und Akzele rometermessung im Vergleich ein Beitrag zur Validierung des MoMo Aktivitätsfragebogens. Deutsche Zeitschrift für Sportmedizin, 62, 3641.

Kowalski, K. C., Crocker, P. R. E. \& Kowalski, N. P. (1997). Convergent Validity of the Physical Activity Questionnaire for Adolescents. Pediatric Exercise Science, 9, 342352.

Landis, J. R. \& Koch, G. G. (1977). The measurement of observer agreement for categorical data. Biometrics, 33, 159174.

Malina, R.M. (2001). Adherence to physical activity from childhood to adulthood: A perspective from tracking studies. Quest, 53, 346355.

Matthews, C. E. (2002). Use of Self Report Instruments to As sess Physical Activity. In G. J. Welk (Ed.), Physical Activity Assessments for Health Related Research (pp. 107 123) Champaign, IL: Human Kinetics.

Neumann, P. (1997). „Zeit eilt, weilt und heilt“. Eine pädago gische Suche nach unterstützenden Zeiterfahrungen im Sport. Spectrum der Sportwissenschaften, 9 (2), 2030

Pate, R. R., Saunders, R. P., Ward, D. S., Felton, G., Trost, S. G. \& Dowda, M. (2003) Evaluation of a community based in tervention to promote physical activity in youth: Lessons from Active Winners. American Journal of Health Promo tion, 17, 171182.

Pate, R. R., Ward, D. S., Saunders, R. P., Felton, G., Dishman, R. K. \& Dowda, M. (2005). Promotion of physical activity among high school girls: a randomized controlled trial. American Journal of Public Health, 95, 15821587.

Prochaska, J. J., Sallis, J. F. \& Long, B. (2001). A Physical Activity Screening Measure for Use With Adolescents in Primary Care. Archives of Pediatrics \& Adolescent Medi cine, 155, 554559.

Schwarz, N. \& Oyserman, D. (2001). Asking questions about be haviour: Cognition, communication und questionnaire con struction. American Journal of Evaluation, 22, 127160.

Shrout, P. E., \& Fleiss, J. L. (1979). Intraclass correlations: uses in assessing rater reliability. Psychological Bulletin, 36, 420428 .

Strong, W. B., Malina, R. M., Blimkie, C. J., Daniels, S. R., Dishman, R. K., Gutin, B. et al. (2005). Evidence based physical activity for school age youth. Journal of pediatrics, 146, 732737

Telford, A., Salmon, J., Jolley, D. \& Crawford, J. (2004). Reli ability and Validity of Physical Activity Questionnaires for Children: The Children's Leisure Activities Study Survey. Pediatric Exercise Science, 16, 6478.

Treuth, M. S., Hou, N., Young, D. R. \& Mayland, L. M. (2005). Validity and Reliability of the Fels Physical Activity Ques tionnaire for Children. Medicine and Science in Sports and Exercise, 37, 448495.

Troiano, R. P., Berrigan, D., Dodd, K. W., Masse, L. C., Tilert, T. \& McDowell, M. (2008). Physical Activity in the United States measured by accelerometer. Medicine and Science in Sports and Exercise, 40, 181188.
Troped, P. J., Wiecha, J. L., Fragala, M., Matthews, C. E., Finkel stein, D. M., Kim, J. et al. (2007). Reliability and validity of YRBS physical activity items among middle school students. Medicine and Science in Sports Exercise, 39, 416425.

Trost, S. G. (2007). Measurement of Physical Activity in Chil dren and Adolescents. American Journal of Lifestyle Medi cine, 4, 299314

Trost, S. G., Ward D. S., McGraw B. \& Pate R. R. (1999). Validity of the Previous Day Physical Activity Recall (PDPAR) in fifth grade children. Pediatric Exercise Science, 11, 341348.

Trost, S. G., Ward, D. S., Moorehead, S. M., Watson, P. D., Riner, W. \& Burke, J. R. (1998). Validity of the Computer Science and Applications (CSA) activity monitor in children. Medi cine and Science in Sports and Exercise, 30, 629633.

Völker, K. (2009). Wie Bewegung und Sport zur Gesundheit beitragen Tracking Pfade von Bewegung und Sport zur Gesundheit. In W. Schmidt (Hrsg.), Zweiter Deutscher Kinder und Jugendsportbericht. Schwerpunkt: Kindheit (S. 89 106). Schorndorf: Hofmann.

Watkinson, C., Sluijs, E. M. F., Sutton, S., Hardeman, W., Cor der, K. \& Griffin, S. J. (2010). Overestimation of physical activity level is associated with lower BMI: a cross sectional analysis. International Journal of Behavioral Nutrition and Physical Activity, 7 (1), 68.

Welk, G. J. (2002). Physical Activity Assessments for Health Related Research. Champaign, IL: Human Kinetics.

West Suitor, C. \& Kraak, V. I. (2007). Adequacy of evidence for physical activity guidelines development: workshop sum mary. Washington, D.C.: The National Academies Press.

Weston, A. T., Petosa R. \& Pate R. R. (1997). Validation of an instrument for measurement of physical activity in youth. Medicine and Science in Sports Exercise, 29, 138143.

WHO, World Health Organisation (2010). Global Recommen dations on Physical Activity for Health. Geneva: WHO.

Woll, A. (2004). Diagnose körperlich sportlicher Aktivität im Er wachsenenalter. Zeitschrift für Sportpsychologie, 11, 5470.

Wong, S. L., Leatherdale, S. T. \& Manske, S. R. (2006). Reli ability and Validity of a School Based Physical Activity Questionnaire. Medicine and Science in Sports and Exercise, 38,15931600 .

Dr. Darko Jekauc

Prof. Dr. Alexander Woll

Universität Konstanz

Fachgruppe Sportwissenschaft

78457 Konstanz

E Mail: darko.jekauc@uni konstanz.de

Dr. Matthias Wagner

Abteilung für Sport und Bewegung

Fakultät I Institut für Gesundheitswissenschaften

Pädagogische Hochschule Schwäbisch Gmünd

Oberbettringerstraße 200

73525 Schwäbisch Gmünd

Daniela Kahlert

Universität Potsdam

Exzellenzbereich, Kognitionswissenschaft

Professur für Sportpsychologie

Am Neuen Palais 10

14469 Potsdam 